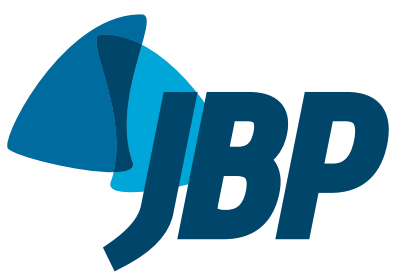

\title{
Angiosarcoma of the lung
}

Mónica Grafino', Paula Alves ${ }^{1}$, Margarida Mendes de Almeida², Patrícia Garrido', Direndra Hasmucrai', Encarnação Teixeira', Renato Sotto-Mayor ${ }^{1}$

1. Departamento do Tórax, Serviço de Pneumologia, Hospital de Dia de Pneumologia Oncológica, Centro Hospitalar Lisboa Norte, EPE, Lisboa, Portugal.

2. Serviço de Anatomia Patológica, Centro Hospitalar Lisboa Norte, EPE, Lisboa, Portugal.

Submitted: 31 August 2015.

Accepted: 18 December 2015.

Study carried out in the Centro Hospitalar Lisboa Norte, EPE, Lisboa, Portugal.

\section{INTRODUCTION}

Angiosarcoma is a malignant endothelial cell tumor of vascular or lymphatic origin and accounts for approximately $2 \%$ of all soft tissue sarcomas. ${ }^{(1)}$ The most common primary sites are the skin and the subcutaneous tissue of the head and neck. ${ }^{(2)}$ Pulmonary involvement is almost always metastatic. ${ }^{(3)}$ Primary pulmonary angiosarcoma is extremely rare, only a few cases having been reported.

\section{CASE REPORT}

A 78-year-old female nonsmoker had previously been examined, a CT scan of the chest having shown a left upper lobe pulmonary mass (Figure $1 \mathrm{~A}$ ). At that time, she underwent bronchoscopy with BAL and bronchial biopsy. The BAL fluid and the biopsy sample were both negative for neoplastic tissue. She subsequently dropped out of follow-up. Two years later, she was referred to our hospital with a six-month history of shortness of breath, dry cough, and weight loss. A chest $X$-ray and a chest CT scan (without contrast) identified a $10 \times 7 \mathrm{~cm}$ lesion in the left upper lobe without forming cleavage plans with the aorta and the pulmonary artery (Figures $1 B$ and $1 C$ ), with a small left pleural effusion and small mediastinal lymph nodes. An ${ }^{18} \mathrm{~F}$-fluorodeoxyglucose positron emission tomography-CT (FDG PET-CT) scan (Figure 1D) showed increased metabolic activity in many organs, greatest in the mass in the upper left lobe, the maximum standardized uptake value (SUVmax) of which was 22. Metabolic activity was also increased in the mediastinal lymph nodes (SUVmax $=7$ ), as well as in the right and left suprarenal glands (SUVmax $=16$ for both); the aortic lumbar lymph nodes (SUVmax = 11); and the right inguinal lymph node (SUVmax = 10). In addition, there was a mesenteric focus, together with subcutaneous tissue nodes and multiple locations in bone.
The bronchoscopic examination revealed a mass occluding the left upper lobe bronchus (Figure 2), and a bronchoscopic biopsy was performed. Before the results of the tissue sample examination had been obtained, she developed right hemiparesis and a new subcutaneous nodule arose. A CT scan showed multiple brain lesions. She was started on corticosteroids. However, she showed no clinical improvement and brain radiotherapy was proposed.

The histological examination of the biopsy specimen showed a soft-tissue neoplasm with a sheet-like or enclosing cleft arrangement of spindle and epithelioid cells with prominent nucleoli, with some multinucleation; mitotic figures were conspicuous, as were extensive necrosis and hemorrhage (Figure $3 \mathrm{~A}$ ). Immunohistochemical staining of the tumor specimen revealed that it was strongly positive for CD31 (Figure 3B), factor VIII-related antigen (Figure 3C), and vimentin; weakly positive for the nuclear transcription factor Fli-1 and cytokeratin AE1/AE3; and negative for CD34, desmin, and smooth muscle actin. Therefore, the histology and immunohistochemistry were both consistent with epithelioid angiosarcoma.

Two weeks after the histologic diagnosis had been made, the patient died. Her death was attributed to the progression of the disease, the brain metastases in particular.

\section{DISCUSSION}

Angiosarcoma in the lung usually represents metastasis from another primary site. Nevertheless, we believe that the case presented here was one of primary pulmonary angiosarcoma. The patient had a two-year evolution of a pulmonary mass that had gone unmonitored and untreated for more than two years. It seems apparent that the previous lesion corresponded to the pulmonary angiosarcoma that evolved and disseminated during that

Correspondence to:

Mónica de Jesus Marques Grafino. Centro Hospitalar Lisboa Norte, EPE; Hospital Pulido Valente, Hospital de Dia de Pneumologia Oncológica, Alameda das Linhas de Torres, 117, 1769-001, Lisboa, Portugal.

Tel.: 35196 1228496. E-mail: mgrafino@gmail.com

Financial support: None. 

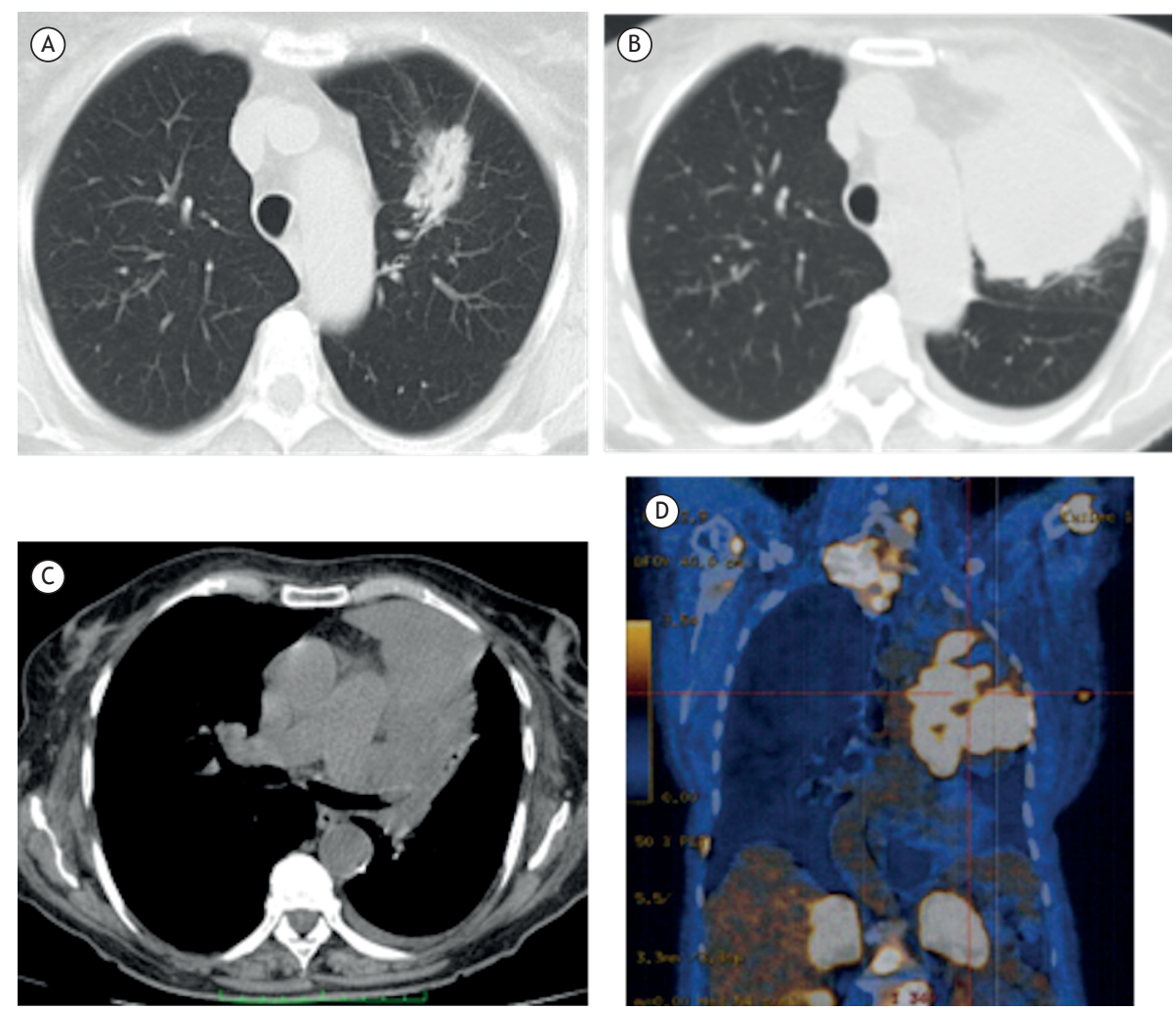

Figure 1. Evolution of angiosarcoma in a 78-year-old female nonsmoker: in A, initial CT scan of the chest showing a left upper lobe pulmonary mass; in B and C, CT scans of the chest, obtained two years later, showing a $10 \times 7 \mathrm{~cm}$ lesion in the left upper lobe, with a small left pleural effusion and small mediastinal lymph nodes; in $\mathrm{D}$, an ${ }^{18} \mathrm{~F}$-fluorodeoxyglucose positron emission tomography-CT scan showing increased metabolic activity in many organs, greatest in the mass in the upper left lobe.

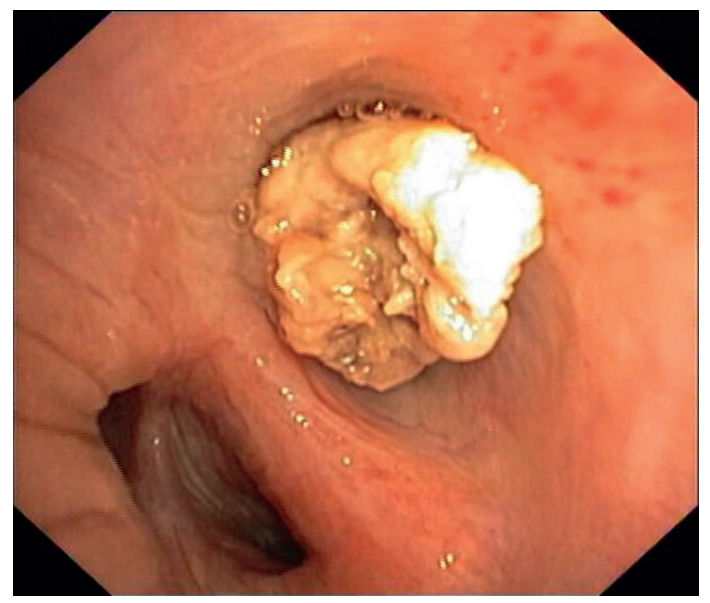

Figure 2. Photograph of a mass occluding the left upper lobe bronchus.

time. This case report also documents the behavior of a primary pulmonary angiosarcoma, which is characterized by insidious growth with extensive local invasion and hematogenous metastasis. ${ }^{(4)}$

Early diagnosis of pulmonary angiosarcoma is not common, because of its rarity and the low index of suspicion. According to data in the literature, the average age of pulmonary angiosarcoma patients is 55.9 years (range, $23.0-82.0$ years) and males are more often affected than are female. ${ }^{(5)}$ Patients usually present nonspecific respiratory symptoms that include hemoptysis, cough, dyspnea, chest pain, and weight loss. On CT scans, primary angiosarcoma can be multifocal or can manifest as solitary lesions. $(4,6,7)$ Imaging with PET can be useful in the diagnosis, staging, ${ }^{(8)}$ and follow-up of patients with angiosarcoma. ${ }^{(9)}$ In the case presented here, the FDG PET-CT scan confirmed its usefulness in identifying the regions and systems involved.

Biopsy and immunohistochemical analysis are essential for the diagnosis of angiosarcoma. The histological features can vary within and among cases. Abnormal, pleomorphic, malignant endothelial cells constitute the hallmark of angiosarcoma. Those cells can be rounded, polygonal, or fusiform, with or without an epithelioid appearance. In well-differentiated areas, abnormal endothelial cells form functioning vascular sinusoidal channels that are continuous with normal vascular channels. In patients with progressively more aggressive disease, the architecture becomes more chaotic, with less clearly defined vascular spaces. In poorly differentiated areas, the malignant endothelial cells form continuous monolayers, usually with an epithelioid morphology. An angiosarcoma typically expresses endothelial markers including factor VIII-related antigen, CD34, CD31, and Fli-1. Among those markers, factor VIII-related antigen is 

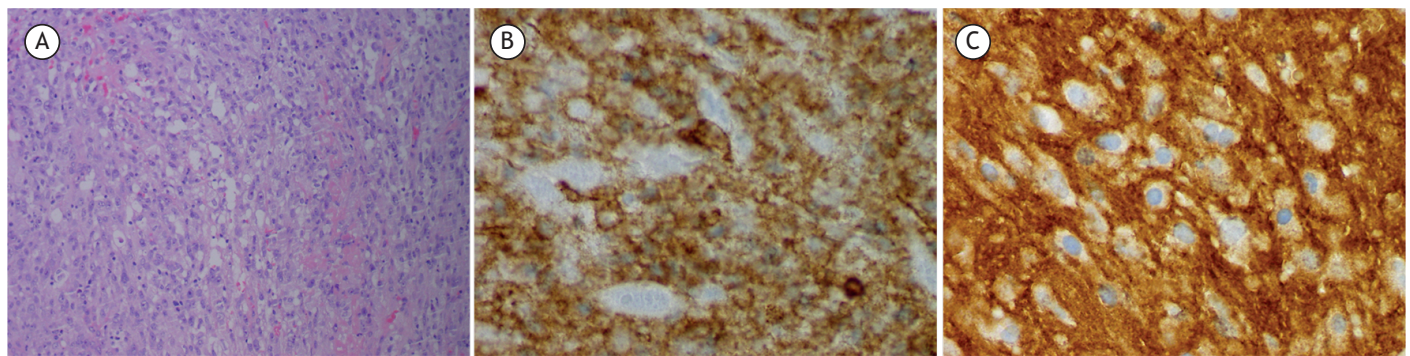

Figure 3. Histological examination of the biopsy specimen: in $A$, extensive necrosis and hemorrhage; in $B$, immunohistochemical staining for CD31; in C, immunohistochemical staining for factor VIII-related antigen.

the most specific but the least sensitive. Positivity for CD31 is relatively specific and extremely sensitive, being detected in approximately $90 \%$ of the cases. In approximately $30 \%$ of the cases, angiosarcoma expresses cytokeratin. ${ }^{(10)}$

Due to the rarity of angiosarcoma, no standardized therapy regimen has been established, especially for primary pulmonary angiosarcoma. Patients with pulmonary angiosarcoma have been treated with radiotherapy, ${ }^{(9,11)}$ surgical resection, ${ }^{(4,9)}$ immunotherapy ${ }_{r}^{(4,6,11)}$ chemotherapy, ${ }_{r}^{(4,6,9)}$ or a combination of those. ${ }^{(4,6,9,11)}$ Although none of those treatments have been shown to be effective, there have been reports of effective treatments for the condition, ${ }^{(4,9,11)}$ especially in cases of localized disease. It has been proposed that the most effective treatment is surgery, which should be considered as early as possible for resectable tumors. ${ }^{(5)}$ However, neoplasms are often inoperable at the time of diagnosis. Multimodality therapy, such as the combination of radiotherapy and immunotherapy (recombinant interleukin-2)(11) and that of surgery and chemotherapy, ${ }^{(4)}$ has also proven to be effective. Chemotherapy with gemcitabine and docetaxel has been reported to produce a complete radiographic response..$^{(9)}$

The prognosis of pulmonary angiosarcoma is poor, mortality within the first few months after clinical presentation, as occurred in the case presented here, approaching $100 \%$. $^{(4-7)}$

Angiosarcoma is a rare malignant neoplasm, and only a few dozen cases of primary pulmonary angiosarcoma have been reported in the literature. It is a diagnostic challenge for clinicians and pathologists. A high index of suspicion is essential to its early diagnosis and could improve its poor prognosis.

\section{REFERENCES}

1. Young RJ, Brown NJ, Reed MW, Hughes D, Woll PJ. Angiosarcoma. Lancet Oncol. 2010;11(10):983-91. http://dx.doi.org/10.1016/S1470 2045(10)70023-1

2. Penel N, Marréaud S, Robin YM, Hohenberger P. Angiosarcoma: state of the art and perspectives. Crit Rev Oncol Hematol. 2011;80(2):25763. http://dx.doi.org/10.1016//.critrevonc.2010.10.007

3. PatelAM, Ryu JH. Angiosarcoma in the lung. Chest. 1993;103(5):1531 5. http://dx.doi.org/10.1378/chest.103.5.1531

4. Chen YB, Guo LC, Yang L, Feng $W$, Zhang $X Q$, Ling $C H$, et al Angiosarcoma of the lung: 2 cases report and literature reviewed. Lung Cancer. 2010;70(3):352-6. http://dx.doi.org/10.1016/j. lungcan.2010.09.002

5. Shimabukuro I, Yatera K, Noguchi S, Kawanami Y, Iwanami T Nishida C, et al. Primary Pulmonary Angiosarcoma Presenting with Hemoptysis and Ground-Glass Opacity: A Case Report and Literature Review. Tohoku J Exp Med. 2015;237(4):273-8. http://dx.doi. org/10.1620/tjem.237.273

6. Judy BF, Predina JD, Mittal J, Deshpande C, Singhal S. Metastatic Primary Pulmonary Angiosarcoma. Surg Sci. 2011;2(3):130-3. http:// dx.doi.org/10.4236/ss.2011.23026
7. Ozcelik C, Onat S, Yaldiz M, Ozcelik Z. Primary epithelioid angiosarcoma of the lung presenting as pulmonary hemorrhage. Asian Cardiovasc Thorac Ann. 2006;14(1):69-71. http://dx.doi. org/10.1177/021849230601400118

8. Treglia G, Cardillo G, Graziano P. A rare case of primary pulmonary epithelioid angiosarcoma detected by (18)F-FDG PET/ CT. Clin Nucl Med. 2014;39(5):450-2. http://dx.doi.org/10.1097/ RLU.0b013e318292f3b3

9. Wilson R, Glaros S, Brown RK, Michael C, Reisman D. Complete radiographic response of primary pulmonary angiosarcomas following gemcitabine and taxotere. Lung Cancer. 2008;61(1):131-6. http://dx.doi.org/10.1016/j.lungcan.2007.12.006

10. Travis WD, Brambilla E, Muller-Hermelink HK, Harris CC, editors World Health Organization Classification of Tumours. Pathology and Genetics of Tumours of the Lung, Pleura, Thymus and Heart. IARC Press: Lyon; 2004

11. Kojima K, Okamoto I, Ushijima S, Yoshinaga T, Kitaoka M, Suga M, et al. Successful treatment of primary pulmonary angiosarcoma. Chest. 2003;124(6):2397-2400. http://dx.doi.org/10.1378/chest.124.6.2397 